\section{'AC Harostar' Apricot}

\author{
Richard E.C. Layne ${ }^{1}$ and David M. Hunter ${ }^{2}$ \\ Agriculture and Agri-Food Canada, Harrow, Ontario NOR 1G0, Canada
}

Additional index words. Prunus armeniaca, fruit breeding, cold hardiness, disease resistance

'AC Harostar' apricot (Prunus armeniaca L.) is a mid-season apricot released for the Ontario fresh market in 2000. The tree is cold hardy, disease resistant, and bears very attractive and good quality fruit for the fresh market. 'AC Harostar' was tested as HW436 by members of the former Western Ontario Fruit Testing Association [WOFTA, now the Ontario Fruit Testing Association (OFTA)] in regional trials in southern Ontario, Canada, and by members of the former New York State Fruit Testing Cooperative Association (NYFSTCA), and it appears to be adapted to regions where 'Goldcot' and 'Veecot' are successfully grown. This new cultivar, developed by Agriculture and Agri-Food Canada at Harrow, Ontario, Canada (AAFC-Harrow), is recommended by the Ontario Tender Fruit Producers' Marketing Board (OTFPMB) for trial planting in Ontario. 'AC Harostar' has also been tested in the Rhône Valley, France, by Star Fruits. 'AC Harostar', together with 'AC Haroblush' (Layne and Hunter, 2003a) and 'AC Harojoy' (Layne and Hunter, 2003b), are the latest introductions from the AAFC apricot breeding program formerly located at Harrow.

\section{Origin}

'AC Harostar' was selected from a controlled cross whose identity was inadvertently lost, and testing in advanced trials at AAFC-Harrow began in 1976. 'AC Harostar' was propagated on 'Haggith' apricot seedling rootstock (Layne and Harrison, 1975) beginning in 1976. 'ACHarostar'/Haggith was placed in evaluation orchards at AAFC-Harrow in 1986 and compared with 'Veecot', 'AC Haroblush', and 'Harogem' which ripen within a few days of each other in the mid-season. Trees were propagated in cooperation with WOFTA under the designation HW436 and placed in regional trials with WOFTAmembers beginning in 1980. 'AC Harostar' is genetically stable and uniform.

Received for publication 2 Jan. 2002. Accepted for publication 19 May 2002. We thank H.O. Jackson, F.D. Stroud, E.W. Lamoure, M.D. St. Pierre, M.F. Gadsby, and D.B. Jessop for excellent technical assistance. We appreciate the assistance of the Western Ontario Fruit Testing Association and its growermembers in field performance evaluations.

${ }^{1}$ Retired.

${ }^{2}$ To whom reprint requests should be addressed. Present address: Univ. of Guelph, Dept. of Plant Agriculture, 4890 Victoria Ave. North, P.O. Box 7000, Vineland Station, Ontario LOR 2E0 Canada. E-mail: dhunter@uoguelph.ca

\section{Description}

Trees of 'AC Harostar' are upright and of medium vigor. Young shoots have medium anthocyanin coloration on the tips. One-year-old shoots have very few laterals, and a medium number of moderately conspicuous lenticels. Leaves are large and medium green on the upper surface. The shape of the leaf base is truncate, the leaf tip is acuminate, and the angle of the tip is broadly acute. Leaf margins are biserrate and there is a slight undulation of the anin coloration of the upper surface, medium anthocyanin coloration of the lower surface, and typically there are three, large, globose, petiolar glands. In a sample of 30 leaves taken from the mid-point of 30 randomly collected shoots in mid-summer of 1995, leaf blades were $85.0 \pm 7.1 \mathrm{~mm}$ long and $72.6 \pm 5.8 \mathrm{~mm}$ wide, and leaf petioles were $33.7 \pm 3.7 \mathrm{~mm}$ long and $1.7 \pm 0.2 \mathrm{~mm}$ thick.

Flower buds are borne on spurs and on 1 -year-old shoots. Flower bud density on 1 -year-old shoots is medium. Flowers are medium size, with white petals when fully open. The petals are circular in shape with a claw of medium length. The stigma is at the same level as the anthers.

Fruits of 'AC Harostar' (Fig. 1) are large, oblong in profile view, trapezoidal in frontal view, predominantly symmetric along the suture, which is shallow. The pedicel cavity is of leaf margin. Leaf petioles have strong anthocy-

Fig. 1. Fruits of 'AC Harostar' apricot (scale in centimeters). medium depth, and the tip of the fruit is rounded with a trace of stylar tissue present at the tip. On tree-ripe fruits, fuzz is inconspicuous and sparse, and the skin surface appears smooth to the naked eye. The ground color of tree-ripe fruits is orange and a bright, solid red blush covers $\approx 50 \%$ of the fruit surface. In 1995, in a random sample of 30 firm ripe fruit, the fruit length was $55.2 \pm 2.7 \mathrm{~mm}$, the cheek width was $49.9 \pm 2.3 \mathrm{~mm}$, and thickness across the suture was $44.1 \pm 2.2 \mathrm{~mm}$. Fruit weight was $64.2 \pm 8.3 \mathrm{~g}$, with stone weight accounting for $5.2 \% \pm 0.5 \%$ of the fruit weight. The flesh is orange, fine textured, medium-firm, and freestone. The stone is oblong, plump, tan in color when dry, and has an acuminate apex, distinct wings and keel, and a grainy surface. Kernels are plump and bitter like 'Veecot', 'AC Haroblush', and 'Harogem', and not sweet like 'AC Harojoy'.

\section{Performance}

In a replicated trial at AAFC-Harrow, 'AC Harostar' was compared from 1988 to 1995 with 'Veecot', 'AC Haroblush', and 'Harogem', which ripen within a few days of each other (Table 1). 'AC Harostar' was less vigorous than 'Veecot', 'AC Haroblush', and 'Harogem'. 'AC Harostar' ranked second in winter hardiness, being less hardy than 'AC Haroblush' but hardier than 'Veecot' and 'Harogem'. 'AC Harostar' ranked second in perennial canker (Leucostoma sp.) resistance, being less resistant than 'AC Haroblush' but more resistant than 'Veecot' and 'Harogem'. Resistance of 'AC Harostar' to bacterial leaf spot [caused by Xanthanomas campestris pv. pruni (Smith) Dye] equaled that of the other cultivars; however, 'AC Harostar' was less resistant than 'AC Haroblush' and 'Veecot' to bacterial fruit spot (also caused by X. campestris pv. pruni), resistance equalling that of

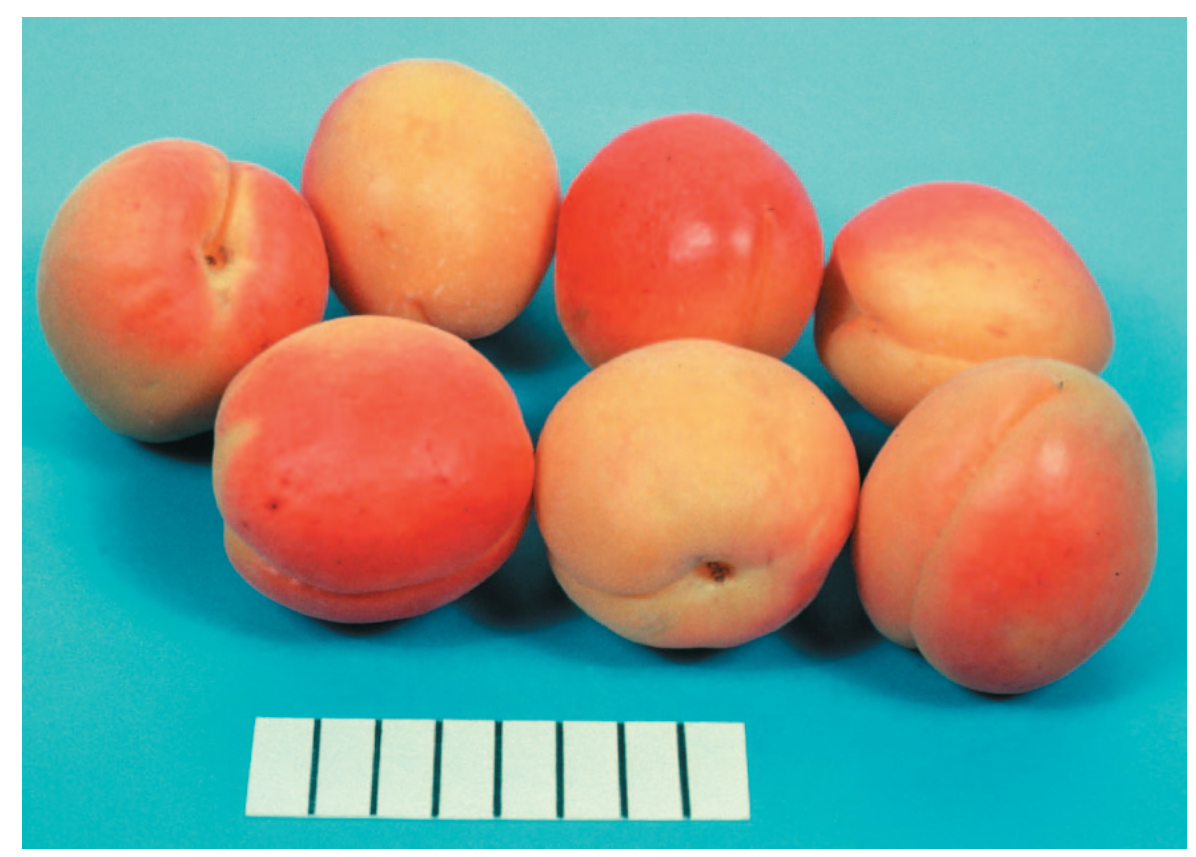


'Harogem'. Productivity of 'AC Harostar' was equal to 'Veecot' and less than 'AC Haroblush' and 'Harogem'.

Fruit size of 'AC Harostar' was greater than 'Veecot' and similar to 'AC Haroblush' and 'Harogem'. 'AC Harostar' was the most attractive of the four cultivars and had similar red blush to 'AC Haroblush' and substantially more than 'Veecot'. Flesh firmness was the same as 'Veecot' and 'Harogem', but greater than 'AC Haroblush'. For all four cultivars, fruit flesh did not adhere to the pit. Fruit flavor of 'AC Harostar' was equal to 'AC Haroblush' and 'Harogem' and superior to 'Veecot'. 'AC Harostar' ranked third in overall performance of the four cultivars and exceeded 'Veecot', the mid-season standard. 'AC Harostar' ripened 23 July at AAFC-Harrow, 1 and $2 \mathrm{~d}$ later, respectively, than 'AC Haroblush' and 'Veecot', and 2 d before 'Harogem'. During the 8-year test period, 'AC Harostar' did not exhibit any major faults, such as skin cracking and preharvest drop. Brown rot [Monilinia fructicola (Wint.) Honey] was present at a very low level in most years and was only a problem in one year (1991), when disease pressure from adjacent trees was high.

Raw product tests carried out in 1995 with pulp of 'AC Harostar' showed that soluble solids content was $12.5{ }^{\circ} \mathrm{Brix}, \mathrm{pH}$ was 3.39 , and titratable acidity (\% citric) was 1.44; corresponding values for 'Veecot', the laboratory standard, were $11.7^{\circ} \mathrm{Brix}, \mathrm{pH} 3.53$, and titratable acidity 1.32 .

In 1990, ripened fruits were processed as canned halves in $40 \%(\mathrm{w} / \mathrm{v})$ syrup and as puree with $20 \%$ (w/w) granulated sugar. In masked identity taste panels, four trained panelists rated canned halves of 'AC Harostar' inferior to 'Veecot' (the laboratory standard), and the overall rating was lowest of the 10 cultivars and advanced selections tested. Thus, 'AC Harostar' is not suitable for home processing as canned halves. In evaluations of the processed puree, 'AC Harostar' was ranked below 'Veecot', but higher than 'Goldcot', the other laboratory standard, and was therefore suitable for this use.

Controlled freezing tests carried out on dormant, fully acclimated shoots using a standard protocol (Layne and Gadsby, 1995) showed that flower buds of 'AC Harostar' were almost as hardy as those of 'Goldcot' (a hardy standard), while shoot xylem was slightly more hardy than 'Goldcot'. The 5-year (1988-91, 1994) average $T_{50}$ for flower buds and shoot xylem of 'AC Harostar' was -28.1 and -36.0 ${ }^{\circ} \mathrm{C}$, respectively. The $\mathrm{T}_{50}$ for 'Goldcot' flower

Table 1. Long-term performance of 'AC Harostar' at Harrow, Ontario, Canada, compared with 'Veecot', 'AC Haroblush', and 'Harogem (1988-95). ${ }^{\mathrm{z}}$

\begin{tabular}{lcccc}
\hline & \multicolumn{3}{c}{ Mean cultivar rating } \\
\cline { 2 - 5 } Characteristics evaluated & Veecot & AC Haroblush & AC Harostar & Harogem \\
\hline Tree vigor & 8 & 8 & 7 & 8 \\
Winter hardiness & 7 & 10 & 9 & 8 \\
Perennial canker & 8 & 10 & 9 & 8 \\
Bacterial leaf spot & 9 & 9 & 9 & 9 \\
Bacterial fruit spot & 8 & 9 & 7 & 7 \\
Productivity & 5 & 6 & 5 & 6 \\
Ripening uniformity & 6 & 7 & 7 & 7 \\
Fruit size & 6 & 7 & 7 & 8 \\
Attractiveness & 7 & 7 & 7 & 5 \\
Blush & 1 & 5 & 4 & 8 \\
Flesh firmness & 8 & 7 & 8 & 7 \\
Flesh adherence to pit & 10 & 10 & 7 & 96 \\
Flavor & 6 & 7 & 94 & 25 July \\
Total score & 89 & 22 July & 23 July \\
Mean ripe date & 21 July & & 7 & 7 \\
Ratings
\end{tabular}

${ }^{2}$ Ratings were subjective on a scale of 1 (least desirable) to 10 (most desirable). Except for blush, ratings of 1 to 4 are considered unacceptable for a commercial cultivar; ratings of 5 and 6 are commercially acceptable; while ratings $\geq 7$ indicate a good to excellent level of performance.

${ }^{y}$ Means for three single-tree replicates planted in a completely random design.

buds and shoot xylem was -29.1 and -35.7 ${ }^{\circ} \mathrm{C}$, respectively. Layne and Gadsby (1995) reported that 'ACHarostar' was in the same bud and shoot xylem hardiness class as 'Goldcot', the hardy commercial standard. Based on these results, 'AC Harostar' should be adapted to the cooler areas in southern Ontario, Canada, and the neighboring U.S. states near the Great Lakes basin, where 'Veecot' and 'Goldcot' are successfully grown.

Based on tree performance, fruit quality, and winter hardiness evaluations, 'AC Harostar', together with 'AC Haroblush' and 'AC Harojoy', which are being introduced at the same time (Layne and Hunter, 2003a, 2003b), will provide growers with additional opportunities for supplying the mid-season apricot market.

\section{Availability}

In testing carried out at the Canadian Food Inspection Agency's Centre for Plant Health, Sidney, B.C. (CPH-Sidney), 'AC Harostar' was found to be free of all known viruses, virus-like agents, viroids, and phytoplasmas, using an internationally approved range of woody and herbaceous host biological indicators, and by serological and molecular methods (D. Thompson, personal communication). Trees propagated from virus-free budwood have been planted in the Canadian Clonal Gene Bank at AAFC-Harrow. In annual monitoring at AAFC-Harrow, these budwood trees have been tested free of prunus necrotic ringspot virus, prune dwarf virus, and tomato ringspot virus using herbaceous (cucumber) and woody ('Shirofugen' cherry) indicators. Limited quantities of virus-tested budwood are available from AAFC-Harrow and CPH-Sidney. Testing of 'AC Harostar' is subject to signing a non-propagation testing agreement with AAFC-Harrow. Information on commercialization licenses in Canada and the United States can be obtained by contacting the Director, Agriculture and Agri-Food Canada, Greenhouse and Processing Crops Research Centre, Harrow, Ontario NOR 1G0, Canada. 'AC Harostar' has been protected in the European Union on behalf of Agriculture and Agri-Food Canada by Star Fruits, Route d'Orange, 84860 Caderousse, France, to whom inquiries for commercial propagation of ' $\mathrm{AC}$ Harostar' in the European Union should be directed.

\section{Literature Cited}

Layne, R.E.C. and M.F. Gadsby. 1995. Determination of cold hardiness and estimation of potential breeding value of apricot germplasm. Fruit Var. J. 49:242-248.

Layne, R.E.C. and T.B. Harrison. 1975. 'Haggith' apricot: Rootstock seed source. HortScience $10: 428$

Layne, R.E.C. and D.M. Hunter. 2003a. 'AC Haroblush' apricot. HortScience 38:142-143.

Layne, R.E.C. and D.M. Hunter. 2003b. 'AC Harojoy' apricot. HortScience 38:138-139. 